\title{
Thickness Distribution of Fresh Eardrums of Cat Obtained with Confocal Microscopy
}

\author{
Liesbeth C. Kuypers, ${ }^{1}$ W. F. Decraemer, ${ }^{1}$ J. J. J. Dirckx, ${ }^{1}$ and J.-P. Timmermans ${ }^{2}$ \\ ${ }^{1}$ Laboratory of Biomedical Physics, Department of Physics, University of Antwerp, Antwerp, Belgium \\ ${ }^{2}$ Laboratory of Cell Biology and Histology, Department of Biomedical Sciences, University of Antwerp, Antwerp, Belgium
}

Received: 7 December 2004; Accepted: 1 April 2005; Online publication: 28 June 2005

\begin{abstract}
The aim of this study was to measure the spatial thickness distribution of the cat tympanic membrane (TM), a very thin, virtually transparent and delicate biological membrane. Axial fluorescence images taken perpendicular through isolated TM were recorded for five different cats using confocal laser scanning microscopy. Thickness was measured on the cross-section of the membranes in the axial images. A correction for focal shift due to refractive-index mismatch was applied. Similar thickness distributions were obtained in all measured samples $(n=9)$. The pars tensa had a rather constant thickness in the central region between the annulus and manubrium. The thickness increased steeply toward the peripheral rim. Thickness was smallest in the inferior region, with values ranging between 5.5 and $9 \mu \mathrm{m}$ in the central part and up to $50 \mu \mathrm{m}$ near the annulus. More superiorly, thickness was slightly higher, up to $20 \mu \mathrm{m}$, between the annulus and manubrium. The anterior part was thicker than the posterior side. These findings are strongly different from a current value in the literature. Our data allow a more precise representation of the eardrum in mathematical models, which are a prerequisite for a better understanding of middle-ear mechanics. The optical sectioning technique of the confocal microscope did not result in any preparation artifacts and was therefore also used to quantify shrinkage due to preparation of histological sections of TMs.
\end{abstract}

Correspondence to: Liesbeth C. Kuypers - Laboratory of Biomedical Physics, Department of Physics - University of Antwerp/Campus Middelheim • Groenenborgerlaan 171 B-2020, Antwerp, Belgium. Telephone: (32) 3-265-3438; fax: (32) 3-265-3318; email: Liesbeth. Kuypers@ua.ac.be
Keywords: thickness, tympanic membrane, pars tensa, confocal fluorescence microscopy

\section{INTRODUCTION}

To understand the mechanisms of hearing, we often resort to mathematical modeling techniques and try to mimic the experimental facts. Due to the complexity of, for example, the middle-ear system, a good understanding of the interplay between the various relevant system parameters (thickness and shape of the tympanic membrane (TM), elasticity, damping, mass and geometry of the ossicles, etc.) is necessary. Data in the literature reveal various examples of middle-ear modeling, comprising a more or less detailed description of the eardrum. Such numerical models can be used to analyze the functioning of the normal eardrum and to predict the effects of surgery or disease. Because many experiments used the cat as an animal model, an extensive body of data on cat hearing is available. Consequently, several cat eardrum model studies have been published (Rabbitt and Holmes 1986, 1988; Funnell et al. 1987, 1992; Funnell 1995; Ladak and Funnell 1995; Funnell and Decraemer 1996; Puria and Allen 1998; Fay et al. 2004).

To obtain realistic results, all mechanical parameters of the eardrum used as input in such a model have to be known accurately. At present, accurate data on the thickness of (TM) are still lacking. We found one direct thickness estimate for the cat eardrum in a histological study on the internal eardrum structure (Lim 1968). The paper reports a thickness range of 30-50 $\mu \mathrm{m}$. Fay et al. (2004) reanalyzed micrographs of two papers in literature (Lim 1968; Chole and 
Kodama 1989) that allow estimating the thickness of a cat (TM) and finding values of 31 and $40 \mu \mathrm{m}$, respectively. In a parallel study, we used the variation of the signal level of a confocal heterodyne interferometer, while it is focused through the membrane (Decraemer et al. 2004). This level peaks when the focus is at the front surface and again when the focus is at the back surface, as such serving as a method to measure thickness. Interpretation of the level versus position curve was not always easy due to, for example, the extreme thinness of the membrane (peak overlap) or excessively high light absorption (ill-defined peaks). The finding that the membrane is extremely thin in certain zones $(\sim 12$ $\mu \mathrm{m})$-i.e., much thinner than what is commonly believed - is confirmed by the present study.

In current mathematical models, the thickness of the cat eardrum is mostly set to a value of $40 \mu \mathrm{m}$ and spatial thickness variations are ignored. Only Fay (2001) and Fay et al. (2004) used a linear taper in thickness ranging from $36 \mu \mathrm{m}$ at the outer edge to 11 $\mu \mathrm{m}$ to the umbo, based on a personal communication of our preliminary results.

Accordingly, only sparse thickness data can be found in the literature for other species (most important studies: cat, Lim 1968; gerbil, von Unge et al. 1991; Teoh et al. 1997; guinea pig, Lim 1968; Igarashi and Kawamata 1993; rat, Magnuson and Hellström 1994; Magnuson et al. 1995, 1997; human, Kojo 1954; Kirikae 1960; Lim 1970; Uebo et al. 1988; Ruah et al. 1991; Funnell and Laszlo 1981; review paper). At best, the thickness was measured at a few points on the eardrum, which hence did not yield a detailed thickness distribution. Most papers do not mention the location on the eardrum surface where the thickness is specified. Furthermore, most measurements were performed with conventional light microscopy or electron microscopy on histological sections of the eardrum. Prior to cutting histological slices of the eardrum, the tissue needed to be fixed and dehydrated (i.e., processes that are known to induce tissue shrinkage), which raises questions on the reliability of the measured thickness values.

This paper describes a method to measure the thickness distribution of an untreated (i.e., without prior dehydration and fixation) eardrum making use of the optical sectioning capability of confocal laser scanning microscopy. Highly accurate thickness values measured along different optical sections of a fresh pars tensa of cat will be presented.

\section{MATERIAL AND METHODS}

\section{Confocal microscopy study}

Material. This report is based on measurements of five right and four left eardrums of five cats (weighing 910,
$1,535,1,970,2,100$, and $3,460 \mathrm{~g})$. The euthanized cats originated from the local shelter. They were sedated and put to sleep first by injecting Myoplegine (Suxamethonii chloridum, Christiaens Pharma, Belgium) and then by administering an intracardiac injection of T61 (Embutramide, Hoechst Roussel Vet, Germany). All ears had pristine eardrums, but one membrane could not be measured because it got torn during preparation.

Specimen preparation. The temporal bone was removed from the skull and a small hole was made in the middle ear cavity wall. The medial surface of the TM was kept moist by applying droplets of phosphate-buffered saline (PBS) to avoid dehydration. The middle ear cavity wall was further removed to fully expose the TM. The malleus was cut at the neck, separating the manubrium from the rest of the ossicular chain. The TM within its fibrocartilaginous annulus was gently dissected out. This sample was stained with Van Gieson dye, a water-based dye that does not cause dehydration or shrinking.

To image the entire TM with the invert confocal laser scanning microscope (CLSM) mounted with an objective lens with a working distance limited to 230 $\mu \mathrm{m}$, the membrane had to be flattened out on the object support, a standard cover glass replacing part of the bottom of a Petri dish (Fig. 1). The membrane could not be spread out perfectly on the glass due to the conical shape of the TM, but folds were avoided

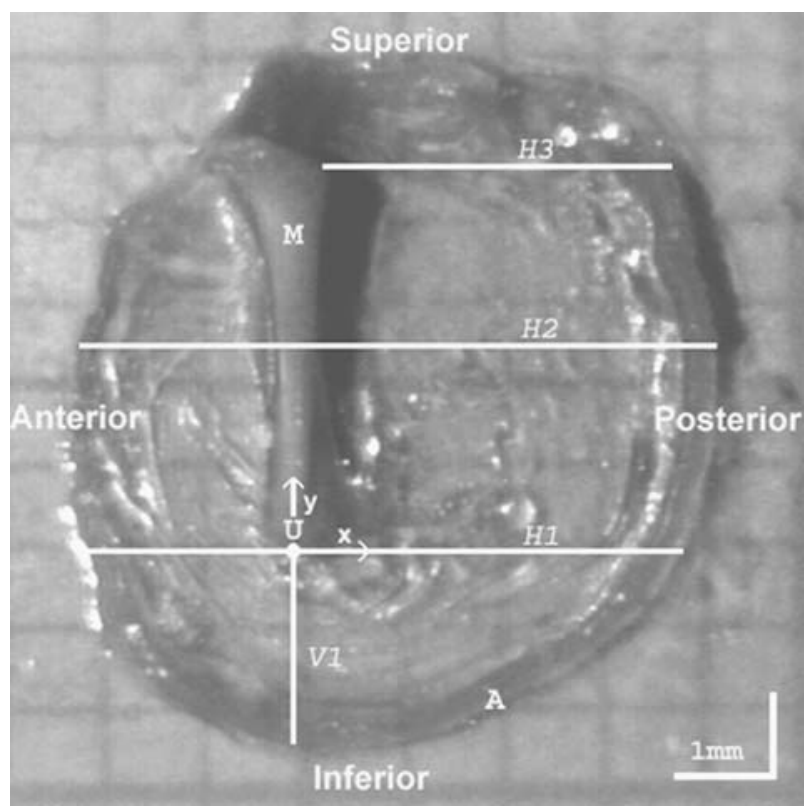

FIG. 1. Right cat eardrum flattened out on a supporting microscope slide with its medial surface facing up ready for measurement with the confocal microscope. A sheet of paper with a 1-mm grid placed underneath served as a scale. Umbo (U), manubrium (M), annulus (A). Also indicated are the lines $\mathrm{V} 1, \mathrm{H} 1, \mathrm{H} 2$, and $\mathrm{H} 3$ where virtual sections are obtained. 
in the regions of interest. The tissue was embedded in glycerin/PBS to avoid desiccation during the long imaging session, which lasted several hours.

Optical sections through the TM. We used a confocal laser scanning microscope (LSM 410 Invert, Zeiss) to obtain axial fluorescence images (i.e., images in a plane parallel to the optical axis) taken perpendicular through the eardrum's surface. The use of a water-immersion objective lens (Zeiss C-Apochromat $40 \times, \mathrm{NA}=1.2$, with correction collar) resulted in axial images with a width of $320 \mu \mathrm{m}$, a theoretical lateral resolution of $0.3 \mu \mathrm{m}$, and a theoretical axial resolution of $0.8 \mu \mathrm{m}$. Scans along a line were preferred over scans over a square area as area scanning substantially prolongs the imaging time and provides only additional information in a very small $320-\mu \mathrm{m}-$ wide band, where thickness variation is marginal. Axial images were measured along several lines, a few millimeters long, through the pars tensa of the eardrum. These lines were obtained by an apposition of a series of about 20 consecutive depth images. The position of each image in the line and the distance between two lines $(x, y$-coordinates in the object plane) were determined by two digital gauges with an accuracy of $1 \mu \mathrm{m}$. Figure 1 shows a dissected right cat eardrum flattened out on the object support as seen from a direction opposite to the microscopic observation direction. The imaged lines are indicated: V1 parallel with the manubrium $(\mathrm{M})$ from the umbo (U) to the annulus (A); H1 perpendicular to the manubrium at the tip (or close to the tip), $\mathrm{H} 2$ at the middle of the manubrium and $\mathrm{H} 3$ at the end of the manubrium. The umbo was chosen as the origin of the $x, y$-coordinate system with the $x$-axis perpendicular to the manubrium and the $y$-axis aligned along the manubrium in the direction of the lateral process. Imaging of a cat eardrum along the four lines took between four and six hours. Systematically imaging the entire eardrum surface (approximately $40 \mathrm{~mm}^{2}$ ) would have been too time-consuming and hence could not guarantee that the tissue is still in a fresh and unaltered condition.

Local thickness measurement. Thickness was measured on the cross-section of the TM shown in an axial image. Figure 2a shows part of such an axial image; the cross-section of the TM is displayed as the white, curved band. The upper surface is the external surface and the lower surface the medial surface. Thickness of the membrane was measured by using the intensity profile taken perpendicular through the cross-section from the upper surface toward the lower surface. A rectangular area with a width of about 20 $\mu \mathrm{m}$ (white rectangle in Fig. 2a) was selected, and the intensity in this selection was laterally averaged to obtain a smooth intensity profile. The resulting intensity profile of the membrane is plotted in

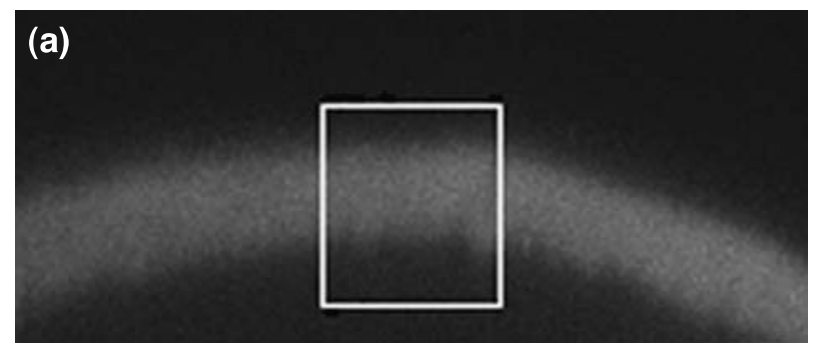

(b)

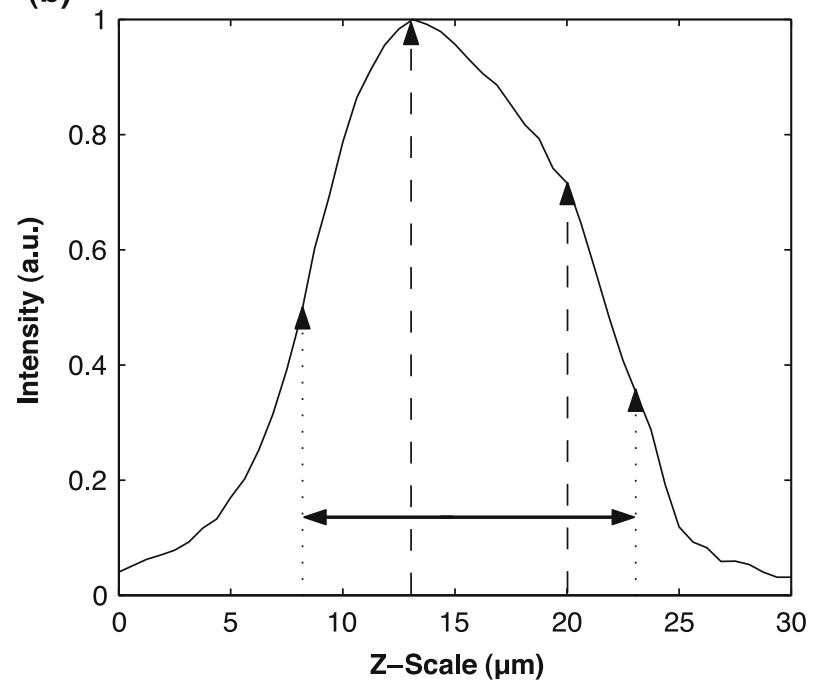

FIG. 2. (a) A small part of an axial image taken perpendicular through the eardrum. The white, curved band shows its crosssection, with the external surface as the upper surface and the medial surface as the lower surface. A selection (shown as a white rectangle) was taken through the cross-section and a laterally averaged intensity profile of the membrane in that selection was obtained, shown in panel b. (b) The intensity profile of the crosssection of the tympanic membrane is shown in panel a. Intensity is plotted as a function of the $z$-coordinate or depth. Dashed arrows: position of the "shoulders" at the start and the end of the slightly decreasing central part. Dotted arrows: position of the half-shoulder intensity. Horizontal arrow: thickness measure.

Figure $2 \mathrm{~b}$. The intensity starts at a very low value (background level) for $z=0 \mu \mathrm{m}$, then rises to a maximum value (in this example, around $z=13 \mu \mathrm{m}$ ) near the upper surface, followed by a segment with a slow decrease in intensity with depth, and finally, near the lower surface, the intensity drops more rapidly (in this example, starting at around $z=20$ $\mu \mathrm{m})$ again to the background level for large $z$-values (in this example, for $z>25 \mu \mathrm{m}$ ). The intensity response profile has an asymmetric ascent and descent and a slowly decreasing middle portion between the left shoulder (first maximum around $z=$ $13 \mu \mathrm{m}$ ) and the right shoulder (around $z=20 \mu \mathrm{m}$ ). All of these features are results of the change in point-spread function with depth, caused by the difference in refractive index $(n)$ between the immersion medium (water) and the specimen (bio- 
logical tissue) (Hell and Stelzer 1995). We have recently shown (Kuypers et al. 2005) that the exact thickness cannot be measured via the commonly used full-width-at-half-maximum (FWHM) criterion. We showed and validated a method to determine the exact thickness in case of such an asymmetric intensity profile, which has also been used in this study. The intensities of the "shoulders" at the start and the end of the gently decreasing central part (indicated with dashed arrows) were determined. The thickness (indicated with solid horizontal arrow) is then determined as the difference between the zposition where half of the left shoulder intensity value (dotted arrow) is found on the ascent and the $z$ position where half of the right shoulder value is found on the descent of the profile.

Refractive index mismatch correction. The refractive index of the immersion medium (water, $n_{1}=1.333$ ) is lower than the refractive index of the specimen (biological tissue, $n_{2} \sim 1.45$ ). It was shown that the thickness of the eardrum in an axial image is consequently smaller by a constant factor than it is in reality. This factor, which is a function of $n_{1}, n_{2}$, and the numerical aperture, can be calculated (Hell and Stelzer 1995; Kuypers et al. 2005). However, we were not able to find a precise value for the refractive index of cat (TM) $\left(n_{2}\right)$ in the literature. Therefore, we have developed an experimental method to determine this correction factor for cases where biological tissues are imaged with an immersion objective lens (Dirckx et al., 2005). To obtain a correction factor for our raw thickness data, such experiments were done on several eardrums, resulting in an average correction factor of $1.095 \pm 0.003$. The thickness of the TM, measured as explained above, is multiplied by this factor to obtain the actual thickness.

Thickness of the TM along the scanning lines. To determine the thickness as a function of the position along the lines scanned on the TM, an interactive computer program in Matlab (The MathWorks, Inc.) was written. After input of parameters, such as scaling factor, image size, and step size between consecutive images, the images (with the individual 320- $\mu \mathrm{m}$ sections) were all opened and aligned with each other. Figure 3 shows an example of a complete scan along the V1 line. The cross-section running from the umbo to the annulus was divided into four vertically superimposed panels. The cross-section starts near the umbo (U), at the left in the top panel, continues after the break at the right from left to right in the second, third, and fourth panels, and ends at the inferior margin (A) at the right in this bottom panel. Thickness was measured at points spaced by about $30 \mu \mathrm{m}$ (cf. the section "Local thickness measurement"). The parts of the image where the TM is seen as slightly slanted with respect
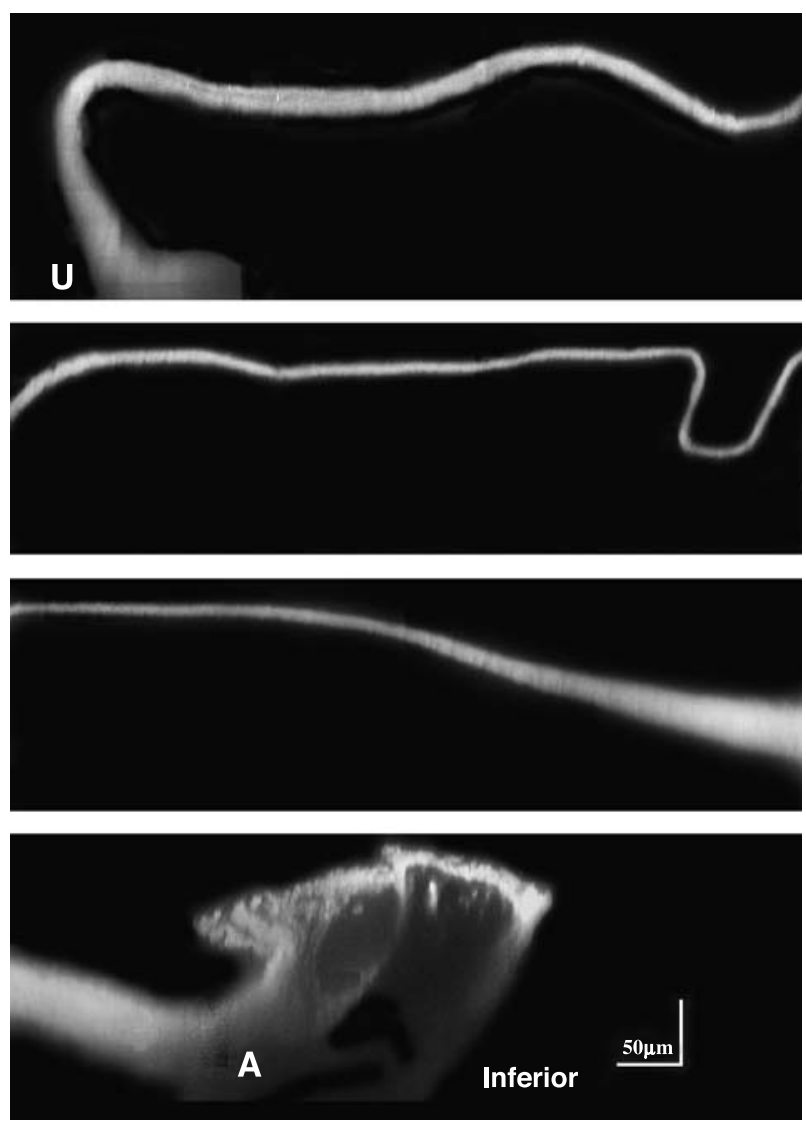

FIG. 3. A complete scan of the cross-section of a right cat tympanic membrane along line $\mathrm{V} 1$. The scan is obtained by aligning nine axial images, each $300 \mu \mathrm{m}$ wide. The scan is divided into four sections that were vertically superimposed. It starts near the umbo $(U)$, at the left in the top panel, continues after the break at the right from left to right in the second, third and fourth panels, and ends at the inferior margin (A) at the right of this bottom panel.

to the horizontal direction were rotated. Because distances in the z-direction have to be corrected (cf. the section "Refractive index mismatch correction"), this rotation can cause an error on the final thickness value. This error is kept below $1 \%$ for rotation angles smaller than $25^{\circ}$. Parts that were strongly slanted due to a wrinkle in the spread-out TM (e.g., in the right part of the second panel in Fig. 3) were not used for measurement. The lateral position within the images was determined by using a calibrated microscale. The position of the image section within the scanning lines was determined using $x, y$ gauges, which indicated the position of the specimen holder on the microscope table.

\section{Conventional light microscopy study}

After completion of confocal imaging, two of the eardrum samples were reused to measure their 
thickness with a conventional light fluorescence microscope to quantify potential thickness differences due to fixation and/or dehydration during the histological preparation process.

Specimen preparation. After washing out the glycerin/PBS by rinsing the tissue in PBS, the TM was further prepared according to a standard procedure for conventional light microscopy. First, it was fixated in $4 \%$ paraformaldehyde, while the membrane was pinned down to maintain its total surface area and to keep it from curling up because of shrinkage, and decalcified in ethylene diamine tetraacetic acid (EDTA) for nine days. After dehydration in a series of ethanol solutions $(70 \%, 80 \%, 90 \%, 100 \%)$, the specimen was kept immersed in methyl benzoate overnight and then in toluene for one hour. The preparation was embedded in paraffin and cut with a microtome (Microm HM $340 \mathrm{E}$ ) into 5- $\mu$ m-thick sections taken perpendicular to the TM surface. One eardrum was cut parallel to the manubrium, and the other perpendicular to the manubrium. Sections made at the positions where the CLSM images were recorded, along the V1 line for the first eardrum and along the $\mathrm{H} 1$ line for the second eardrum, were selected from the stack, making use of the umbo as a landmark. These sections were stained with a hematoxylin-and-eosin solution and mounted on microscope slides.

Imaging and thickness measurement. Two sections, located at lines V1 and H1 (see Fig. 1), were analyzed under a conventional light microscope (BX50, Olympus) using a dry objective lens with $40 \times$ magnification and an NA of 0.85. After length calibration of the image plane, a series of images were recorded. These images were analyzed with a commercial software package (AnalySIS) to measure the TM thickness as the length of line segments taken perpendicular across the TM.

\section{RESULTS}

\section{Thickness along the scanning lines}

In Figure 4, thickness is plotted as a function of the position along the lines $\mathrm{H} 3, \mathrm{H} 2, \mathrm{H} 1$, and $\mathrm{V} 1$ through the left eardrum for one of the cat ears. Figure 5 shows similar plots for the right eardrum of the same cat. Note the difference in scaling between thickness and position. The umbo was chosen as the origin $(x=0, y=0)$ and the manubrium was aligned along the $y$-axis during measurement. The position of line $\mathrm{H} 1$ shown in Figure 4 was selected about $350 \mu \mathrm{m}$ inferior to the umbo to obtain an uninterrupted profile through TM tissue.

The thickness profiles from the peripheral rim toward the manubrium in all plots of Figures 4 and 5
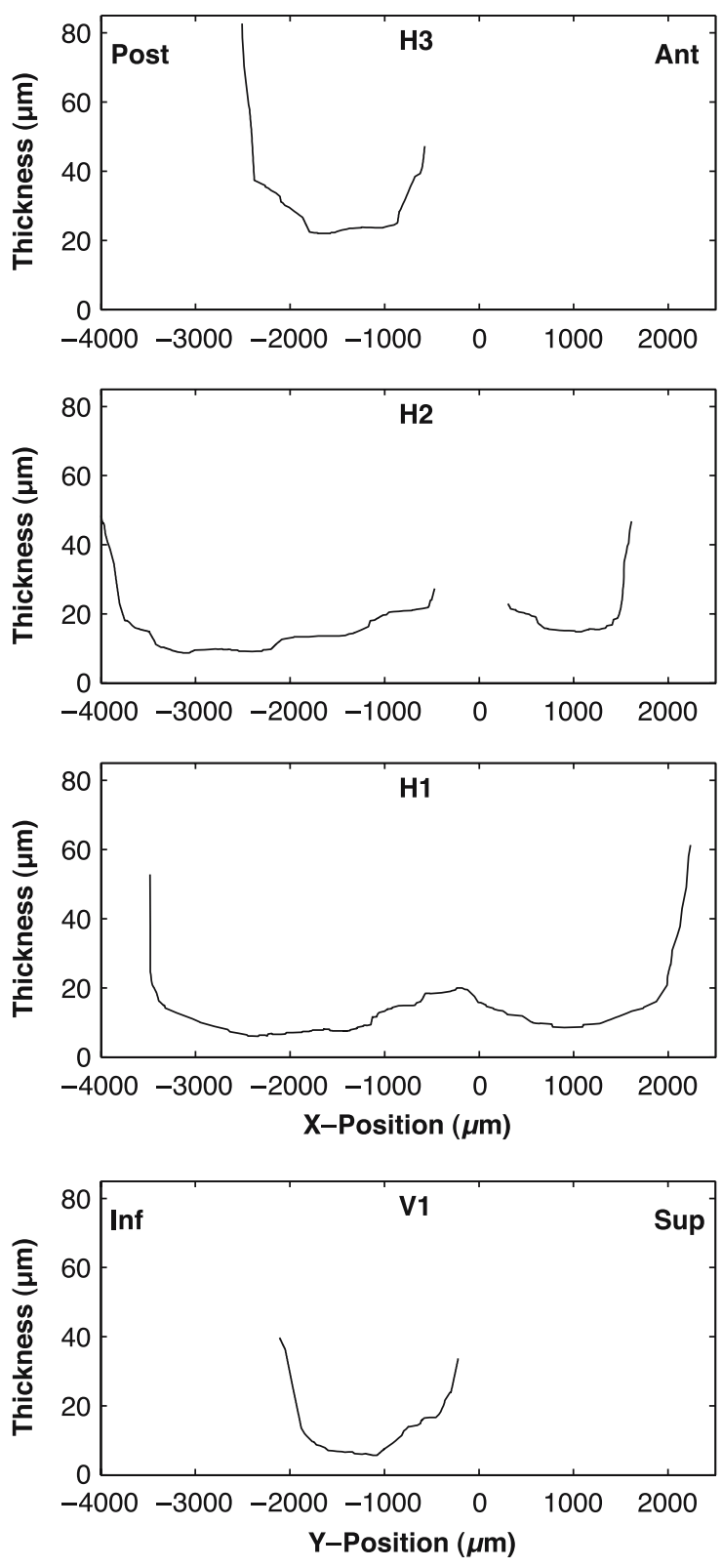

FIG. 4. Thickness as a function of position along the $\mathrm{H} 3, \mathrm{H} 2, \mathrm{H} 1$ and V1 lines for a cat left eardrum. The thickness scale is expanded. The umbo is chosen as the origin. Ant = Anterior, Post = Posterior, Inf $=$ Inferior, Sup = Superior.

show similar features. Thickness is smallest and is rather constant in the central region between the annulus and manubrium. Values in these regions range between 5.5 and $20 \mu \mathrm{m}$. Thickness of the TM increases steeply by a factor of approximately 3 when moving from the central region to the edge of the manubrium. Thickness increases even more, i.e., by a factor close to 4.5 , when moving from the central region toward the rim.

Figures 4 and 5 demonstrate the gradual increase in thickness in the central region from the most inferior region (V1) to the most superior (H3) region. 

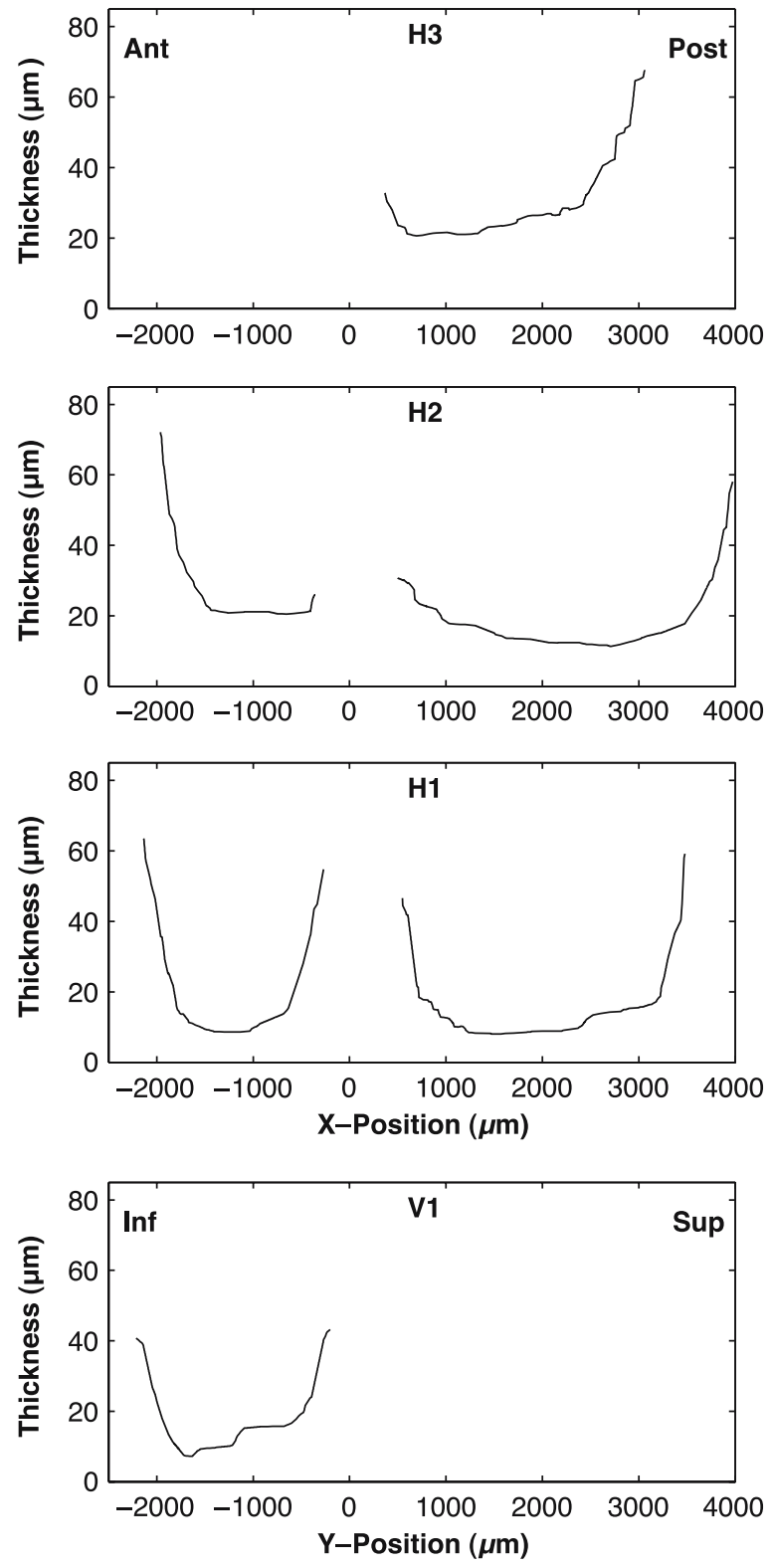

FIG. 5. Thickness as a function of position along the $\mathrm{H} 3, \mathrm{H} 2, \mathrm{H} 1$ and $\mathrm{V} 1$ lines for a right eardrum of the same cat as in Figure 4. The thickness scale is expanded. The umbo is chosen as the origin. Ant = Anterior, Post $=$ Posterior, $\operatorname{Inf}=$ Inferior, Sup $=$ Superior .

Furthermore, the horizontal scan lines H1, H2, and H3 are seen to display the left/right symmetry that can be expected between the left and the right ear of the same animal: values in the symmetric zones are well comparable in the left and right eardrum.

Each cat eardrum was found to exhibit a similar thickness distribution as described in this section. Figure 6 shows thickness profiles along all section lines for four different left eardrums. The profile indicated with the solid line is the same as that shown in Figure 4, positioned slightly more inferior than the other H1 profiles. The features "a central, rather constant, thin region and a steep increase in thickness toward the edges" are found in all eardrums.

\section{Thickness map of the eardrum}

It was not possible to measure the thickness at sufficient points spread across the entire TM to automatically generate a representative thickness map. Using all the measured information (e.g., the TM thickens toward the annulus and the manubrium) and assuming that thickness varies gradually from location to location both radially and circularly, we manually constructed a thickness map by $2 \mathrm{D}$ interpolation of the thickness at many points spread across the major part of the TM. Figure 7 shows this map plotted via a contour plot. The data used originate from the right TM shown in Figure 5. The position of the lines along which measurements were made are indicated. In parts where no measurements were done, the interpolation might not give the exact thickness value, but the regularity and symmetry in this contour plot lead us to believe that the map represents a fairly good representation of the TM thickness distribution.

The plot shows a gradual increase in thickness in the central region from the inferior (V1) to the superior (H3) region. The TM is thinnest $(<12.5 \mu \mathrm{m})$ in the large, central and most inferior regions. The membrane thickens toward the superior side up to $25 \mu \mathrm{m}$ in the anterior side and $30 \mu \mathrm{m}$ in the posterior part.

The thickness variation in the inferior part is approximately symmetrical in the anterior and posterior sides, whereas the thickness distribution becomes gradually more asymmetrical when moving more superiorly. The anterior side is smaller in area but significantly thicker than the posterior side: at midmanubrium height, thickness in the middle of the anterior part is almost twice that in the middle of the posterior part.

\section{Mean thickness values}

Because the thickness along the scanning lines for all measured eardrums was very similar, we derived some typical mean thickness values. First, we averaged the thickness over the central, thinnest region for the lines V1, H1, H2, and H3 for all nine eardrums (see Table 1a). The ratios of the central thickness in $\mathrm{H} 1$, $\mathrm{H} 2$, and $\mathrm{H} 3$ to the thickness in the thinnest part, V1, are given in Table 1b. Midway between manubrium and annulus, the TM has a thickness ranging between 5.7 and $21.1 \mu \mathrm{m}$. The smallest value is found in the posterior-inferior region. The largest thickness is found in the posterior-superior region, which is 2.5 times thicker than that in the central inferior region. 

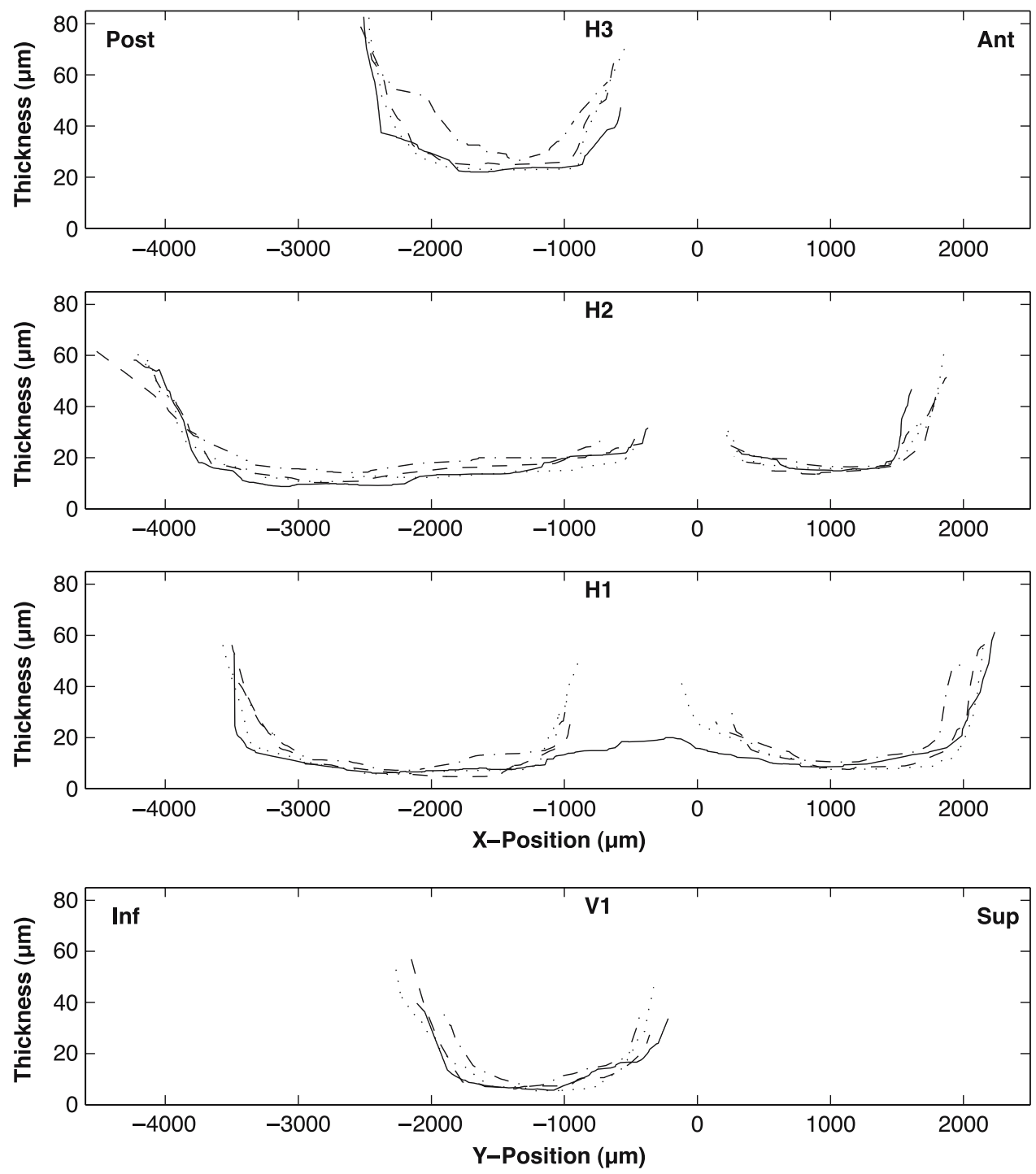

FIG. 6. Thickness as a function of position along the $\mathrm{H} 3, \mathrm{H} 2, \mathrm{H} 1$ and $\mathrm{V} 1$ lines for four different left cat eardrums. The solid-lined profile is the same as that shown in Figure 4, for $\mathrm{H} 1$ positioned more inferior than the other profiles. The thickness scale is expanded. The umbo is chosen as the origin. Ant $=$ Anterior, Post $=$ Posterior, Inf = Inferior, Sup $=$ Superior .

Accordingly, the anterior side is thicker than the posterior side with a ratio of $1.32 \pm 0.13$ along the $\mathrm{H} 1$ line and $1.58 \pm 0.18$ along the $\mathrm{H} 2$ line.

Thickness of the eardrum at the manubrium edge and at the annulus edge shows larger variations. The mean value over all lines and eardrums at the manubrium is $34.7 \pm 1.5 \mu \mathrm{m}$, with a maximal value of $60 \mu \mathrm{m}$. Near the annulus, a mean value of $54.0 \pm$ $2.6 \mu \mathrm{m}$ and a maximal value of $99 \mu \mathrm{m}$ are found. Although the thickness of the TM near the annulus is thicker than that near the manubrium, the slope of the thickening part toward the manubrium is steeper than that toward the annulus by a factor of $1.4 \pm 0.3$.

\section{Thickness obtained with histological sections versus thickness from optical sections}

Figure 8 plots the thickness measured on histological sections as a function of the position along the H1 line and the V1 line (dots). The thickness of untreated tissue obtained with confocal microscopy on the same $\mathrm{H} 1$ and V1 lines is shown on the same plot (solid line). The data along the H1 line are already shown in Figure 6 (dotted line), whereas the data along the V1 line were obtained for a right eardrum that was not been shown yet. The measuring uncertainty in case of the confocal study is $0.9 \mu \mathrm{m}$ for all measured thickness values. For the conventional microscopy study, the error on the data shown is smaller, i.e., $0.21 \mu \mathrm{m}$.

We calculated the mean thickness of the central thin region for both cases. For the H1 line, a mean thickness of $7.2 \mu \mathrm{m}$ for the anterior side and $6.7 \mu \mathrm{m}$ for the posterior side of the fresh membrane was found. Mean thickness values for the same line in the conventional microscopy study were $4.7 \mu \mathrm{m}$ for the anterior side and $5.5 \mu \mathrm{m}$ for the posterior side. Shrinkage of approximately $35 \%$ in the anterior region and $19 \%$ in the posterior region was found. The other membrane shows a mean thickness in the central region along the $\mathrm{V} 1$ line of $9.1 \mu \mathrm{m}$ in fresh 


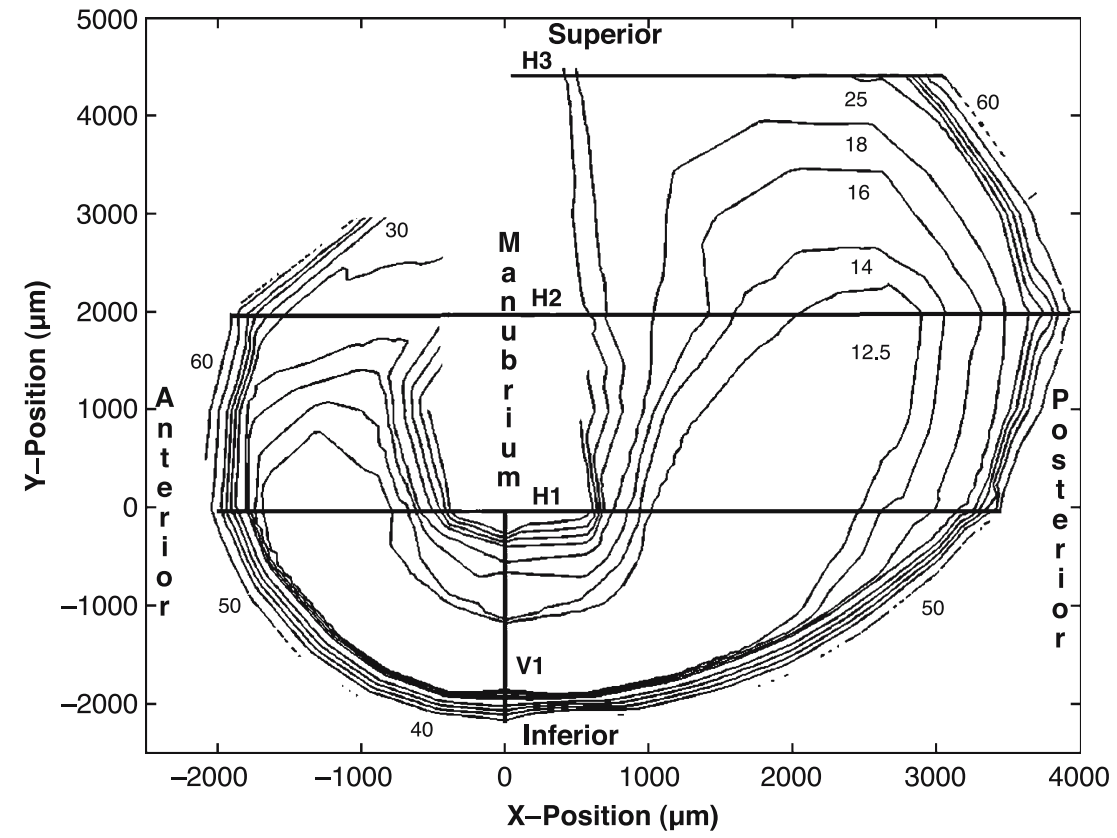

FIG. 7. Contour plot of the thickness distribution of a right cat eardrum (i.e., the same as that shown in Figure 5). Thickness values are in microns $(\mu \mathrm{m})$. The position of the manubrium, and the positions of the lines along which data were obtained, are indicated. condition and a mean value of $7.8 \mu \mathrm{m}$ after histological preparation, which results in a shrinkage of about $16 \%$. Both histologically prepared tissues were found to be thinner than the fresh tissues, with a shrinkage that was significantly larger than the experimental error. An important finding is that shrinkage varies from location to location along the same line.

\section{DISCUSSION}

\section{Thinness of the cat TM}

A most remarkable finding in this study is that the cat (TM) is very thin for the larger part, only about 12 $\mu \mathrm{m}$ thick). Our parallel study using confocal heterodyne interferometry even disclosed values as small as

\section{TABLE 1}

(a) Mean thickness (in $\mu \mathrm{m}$ ) of nine eardrums of the averaged values in the central, thin region between the manubrium and the peripheral rim. (b) Ratio of the data in panel (a) at the different lines with the value of line $\vee 1(8.6 \mu \mathrm{m})$

\begin{tabular}{lrr}
\hline $\begin{array}{l}\text { (a) } \\
\text { Thickness }\end{array}$ & Anterior & \multicolumn{1}{c}{ Posterior } \\
\hline $\mathrm{H} 3$ & & $21.1 \pm 2.1$ \\
$\mathrm{H} 2$ & $17.0 \pm 1.5$ & $10.7 \pm 0.7$ \\
$\mathrm{H} 1$ & $7.5 \pm 0.5$ & $5.7 \pm 0.4$ \\
$\mathrm{~V} 1$ & & \\
(b) & & \\
Ratio & Anterior & Posterior \\
\hline H3/N1 & & $2.5 \pm 0.7$ \\
H2/N1 & & $1.25 \pm 0.14$ \\
H1/N1 & $1.98 \pm 0.24$ & $0.66 \pm 0.07$ \\
\hline
\end{tabular}

$6 \mu \mathrm{m}$ for the cat eardrum. Because both independent methods yield the same extreme thinness, we are confident that these observations are genuine. This finding is also further validated by the Newton thinfilm interference fringes that can sometimes be observed when one looks at a TM under normal white light illumination. Only for extremely thin layers can such fringes be observed.

We measured a thickness of about $12 \mu \mathrm{m}$ in the thinnest region of the TM, which is approximately 3 times smaller than the only thickness value (between 30 and $50 \mu \mathrm{m})$ available in the literature (Lim 1968). We generally found thickness values of about $50 \mu \mathrm{m}$ near the edges, leading us to conclude that the histological sections in the study by Lim were most probably not taken from the central, thinnest region but rather near the edge of the membrane. It is known (Lim 1968) that fiber layers are thick near the edges and thinner in the central region. Because Lim's micrograph of the cross-section of the cat TM (Lim 1968) shows a large amount of fibers and because he further argues that the circular fiber layer becomes very thin near the center, we assume that the thickness measurement was performed at a position near the edge.

\section{Thickness map}

We found that no single value can be given for the thickness of the (TM) of cat. Literature data do not show how thickness varies with position. The thickness map constructed in this study (Fig. 7) shows very clearly that the membrane is thickest around the rim (about $60 \mu \mathrm{m}$ ), becomes gradually thinner when moving inward, remains at an almost constant small 

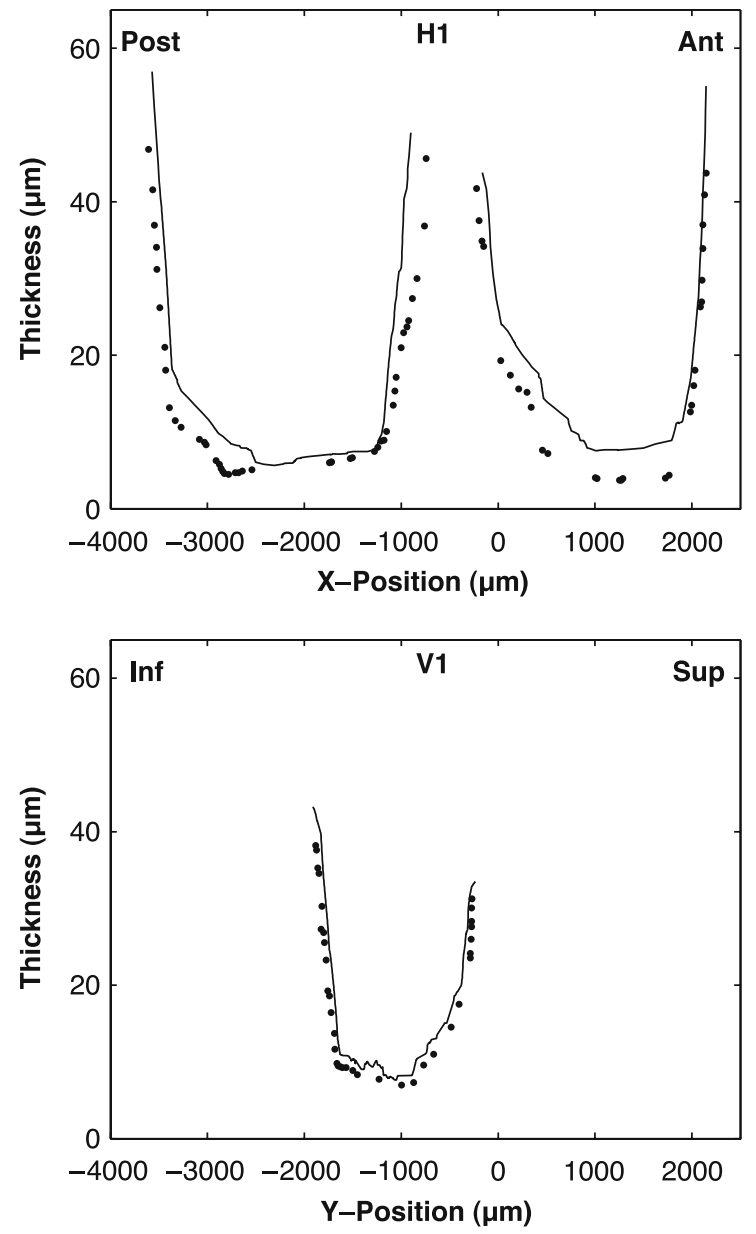

FIG. 8. Thickness as a function of position along the $\mathrm{H} 1$ line and the V1 line. Solid line: fresh tissue with confocal microscopy; dots: prepared tissue with conventional light microscopy. Data for the fresh tissue along the $\mathrm{H} 1$ line are also shown in Figure 6 (dotted line); the data along the V1 line were obtained for a right eardrum. The thickness scale is expanded. The umbo is chosen as the origin. Ant $=$ Anterior, Post $=$ Posterior, $\operatorname{Inf}=$ Inferior, Sup $=$ Superior.

value in the middle zones, and finally thickens again toward the manubrium.

\section{Mass description of the cat TM}

The purpose of the present study was to describe the total thickness of the TM, and as such provide information about its mass distribution. Because the density of the different layers all consist of soft biological material, all layers of the membrane will approximately have equal mass density. Hence the presented total thickness and its local distribution can be reliably used to refine the mass description in existing middle ear models.

Membrane stiffness is mostly provided by the collagenous fibers in the TM. We could not measure the thickness of the circular or radial fiber layers separately, as the layers could not be distinguished in the cross- sections. Micrographs in literature (Lim 1968) show that these layers appear thicker near the manubrium, umbo, and peripheral rim. These findings could suggest that the thicker peripheral parts are also stiffer. However, linking fiber layer thickness directly to stiffness is ill-advised. To obtain the overall TM stiffness, the number of fibers must be combined with individual fiber elasticity and thickness, parameters which still are not sufficiently known to make predictions.

Possible effects of the preparation on the thickness of the TM

As mentioned earlier, the TM consists of several layers that could not be distinguished in the axial fluorescence images taken perpendicular through the TM. To make sure that all the layers were displayed, and thus that the total thickness of the TM was measured, we performed a test. A small piece of the TM was sandwiched between a microscopic glass and a cover glass. The reflection mode of the microscope clearly reveals the surfaces of both glass plates, as well as the fluorescence light emitted by the tissue of the TM. The entire space between the two glass plates was filled with fluorescence light from the TM. These observations confirm that all layers could be seen and that hence the total thickness of the TM was measured.

The TM was removed from the middle ear and thus not kept in its original shape. Any possible preexisting tension in the membrane consequently would have been lost. Could this have influenced its thickness? Let us first state that the existence of pretension of the TM is questioned, since to date no definite proof has been presented for its existence (Funnell and Laszlo 1981; Fay 2001). The difficulty of proving pre-tension suggests that: (1) either there is no such pre-tension, or (2) if it exists, it must be very small. The following numeric example should give an idea about the influence of removing possible pretension on the thickness. In a study on the elasticity of human (TM) by Decraemer et al. (1980, Fig. 3), we see, e.g., that a relative length increase of $3.7 \%$ of a strip of eardrum tissue results in a tension of $4 \mathrm{~N} / \mathrm{m}^{2}$. Such a tension, which is a large overestimate of the potential pre-tension, would result in a relative thickness change of $1.1 \%$ when a Poisson's ratio of 0.3 is used (Funnell and Laszlo 1981). An eardrum with a thickness of $10 \mu \mathrm{m}$ would swell $0.1 \mu \mathrm{m}$ when the pre-tension is removed. Even in this exaggerated case, swelling is highly limited. Thus, pre-tension release, if present at all, can not cause a significant thickening of the membrane.

During imaging, the membrane was embedded in glycerin/PBS to avoid desiccation. Can this embedding change the thickness of the membrane significantly? Thickness change due to PBS can be ruled out 
as it is isotonic. The possible influence of glycerin, which is osmotic and could permeate into the tissue, was investigated. We measured the thickness at consecutive moments along the same scanning line: approx. $1 / 2$ hour, 1 day and 2.5 days after the time of preparation, while it was kept immersed in the fluid. Here, also, no measurable thickness difference, and thus no influence of the glycerin, was observed.

\section{Histological shrinkage}

We found that the preparation method to obtain histological sections induces severe shrinking of the tissue (see "Thickness obtained with histological sections versus thickness from optical sections"). Because the shrinkage along the section is not uniform, it is not possible to determine a single correction factor that could be used to correct the thickness values measured on histological sections of the TM. Therefore, eardrum thickness cannot be reliably measured by using a histological sectioning technique. The method based on confocal microscopy described in this paper can be used on fresh (untreated) material, thus avoiding any shrinkage problems.

Although the actual thickness values obtained with the histological technique are different from the values obtained with the confocal method, they confirm our finding that membrane thickness is strongly dependent on anatomical location and that thickness values much smaller than those cited in literature are found in greater parts of it.

\section{Is there interspecimen variation?}

The thicknesses of distinct eardrums show only slight differences from animal to animal (Fig. 6). One young cat, weighing only $910 \mathrm{~g}$, showed an eardrum (dash-dotted line) that was slightly thicker. It is most probable that the structure and density of this membrane had not yet been developed into the structure of that of the adult cat. The other cats, which exhibited large differences in body weight, but were all grown-up, did not show any relevant thickness differences. We can therefore conclude that the thickness of the adult cat TM does not significantly change from adolescence to old age.

\section{Confocal scanning microscopy method versus} confocal interferometry method

In parallel with this systematic study, we also carried out a smaller study on the thickness measurement of the cat eardrum using a confocal heterodyne interferometer. The advantage of this method lies in the use of confocal scanning microscopy, which directly produces $300-\mu \mathrm{m}$-wide cross-sectional images. The procedure to extract the thickness from the crosssectional profiles is quite straightforward. The interferometric study is a single-point method and determination of the thickness on the section curves (interferometer level versus focusing depth) is not always easy. The interferometer method can be used to its advantage when the membrane is still in place in the temporal bone. No prelevation or flattening out on a glass plate is required. Additionally, it is possible to simultaneously construct the 3D shape of the TM. Both methods have their strong and weak points. Both techniques used in parallel have mutually validated the results found.

\section{CONCLUSION}

We measured with great accuracy the thickness of fresh cat TM based on cross-sectional fluorescence images obtained with a commercial confocal microscope. We also showed that the classical histological preparation causes location-dependent shrinkage and that hence no single valued correction factor exists to correct for shrinkage. Histological measured thickness of the eardrum provides an approximate value that, in fact, is an underestimation.

All nine cat eardrums had similar thickness profiles in all measured parts of the pars tensa: (1) a large thickness was observed at the annulus and manubrium and a very thin central part was seen, (2) the thinnest region was found on the inferior side with a thickness in the order of $10 \mu \mathrm{m}$, (3) the thickest region was found in the superior-posterior region with a thickness in the order of $25 \mu \mathrm{m}$, and (4) the anterior region was significantly thicker than the posterior region.

Using all data and assuming a gradual change in thickness variation in the radial and circumferential directions, we could construct a thickness map for most of the pars tensa. To our knowledge, this is the first time that such precise and complete thickness data for the eardrum of any species are given. Our results advise against the use of single thickness values in mathematical models and, most importantly, show that the cat TM is, for the larger part of its surface, two to three times thinner than the values used in most mathematical models (except for the model of Fay 2001 and Fay et al. 2004) cited in literature.

\section{ACKNOWLEDGMENTS}

The authors wish to thank Jan van Daele and Ria Spillemaeckers for technical assistance. We are also indebted to Dr. vet. Chris Van Ginneken of the Department of 
Veterinary Sciences of the University of Antwerp for her help in providing the specimens. This paper was supported by a grant of the Institute for the Promotion of Innovation through Science and Technology in Flanders (IWT-Vlaanderen)

\section{REFERENCES}

Chole RA, Kodama K. Comparative histology of the tympanic membrane and its relationship to cholesteatoma. Ann. Otol. Rhinol. Laryngol. 98:761-766, 1989.

Decraemer WF, Maes MA, Vanhuyse VJ. An elastic stress-strain relation for soft biological tissues based on a structural model. J. Biomech. 13:463-468, 1980.

Decraemer WF, Khanna SM, Dirckx JJJ. Measurements of the thickness of the tympanic membrane in cat using a heterodyne interferometer. In: Tomasini EP (ed) Vibration Measurements by Laser Techniques: Advances and Applications, vol. 5503, pp 106-115, 2004.

Dirckx JJJ, Kuypers LC, Decraener WF. Refractive index of tissue measured with confocal microscopy. J. Biomed. Opt. in press (2005).

FAY JP. Cat eardrum mechanics. Dissertation. Stanford University, California, 2001

Fay J, Puria S, Decraemer WF, Steele C. Three approaches for estimating the elastic modulus of the tympanic membrane. J. Biomech., available online since November, in press, 2004.

FunNell WRJ. Low-frequency coupling between eardrum and manubrium in a finite-element model. J. Acoust. Soc. Am. 99:3036-3043, 1995.

FunNELl WRJ, Decraemer WF. On the incorporation of moiré shape measurements in finite-element models of the cat eardrum. J. Acoust. Soc. Am. 100:925-932, 1996.

FunNell WRJ, LASzlo CA. A critical review of experimental observations on eardrum structure and function. Otorhinolaryngology 44:181-205, 1981.

Funnell WRJ, Decraemer WF, Khanna SM. On the damped frequency response of a finite-element model of the cat eardrum. J. Acoust. Soc. Am. 81:1851-1859, 1987.

Funnell WRJ, Khanna SM, Decraemer WF. On the degree of rigidity of the manubrium in a finite-element model of the cat eardrum. J. Acoust. Soc. Am. 91:2082-2090, 1992.

Hell SW, Stelzer EHK. Lens aberrations in confocal fluorescence microscopy. In: Pawley JB (eds) Handbook of Biological Confocal Microscopy. Plenum Press. New York, pp 347-354, 1995.

IgARASHI Y, KaWAMATA S. The fine structure of the guinea pig tympanic membrane with special reference to the fiber arrangement. Acta Otolaryngol., Suppl. 504:140-142, 1993.

KIRIKAE I. The Structure and Function of the Middle Ear. University of Tokyo Press. Tokyo, 1960.

Kojo Y. Morphological studies of the human tympanic membrane. J. Otolaryngol. Jpn. 57:115-126, 1954.

Kuypers LC, Decraemer WF, Dirckx JJJ. A procedure to determine the correct thickness of an object with confocal microscopy in case of refractive index mismatch. J. Microsc. 218:68-78, 2005.

LADAK HM, FUnNELl WRJ. On the effects of geometric nonlinearities in a finite-element model of the cat eardrum. In: Proc. IEEE EMBS 17th Annual Conference \& 21st Can. Med. \& Biol. Eng. Conf., Montréal, pp 1439-1440, 1995.

LIM DJ. Tympanic membrane: electron microscopic observation. Part 1. Pars tensa. Acta Otolaryngol. 66:181-198, 1968.

Lim DJ. Human tympanic membrane: an ultrastructural observation. Acta Otolaryngol 70:176-186, 1970.

Magnuson K, Hellström S. Early structural changes in the rat tympanic membrane during pneumococcal otitis media. Eur. Arch. Otorhinolaryngol. 251:393-398, 1994.

Magnuson K, Hellström S, Magnuson B. Structural changes in the rat tympanic membrane following repeated pressure loads. Eur. Arch. Otorhinolaryngol. 252:76-82, 1995.

Magnuson K, Hermansson A, Melhus A, Hellström S. The tympanic membrane and middle ear mucosa during non-typeable Haemophilus influenzae and Haemophilus influenzae type b acute otitis media: a study in the rat. Acta Otolaryngol. 117:396-405, 1997.

Puria S, Allen JB. Measurements and model of the cat middle ear: evidence of tympanic membrane delay. J. Acoust. Soc. Am. 104:3463-3481, 1998.

Rabiitt RD, Holmes MH. A fibrous dynamic continuum model of the tympanic membrane. J. Acoust. Soc. Am. 80:1716-1728, 1986.

Rabitt RD, Holmes MH. Three-dimensional acoustic waves in the ear canal and their interaction with the tympanic membrane. J. Acoust. Soc. Am. 83:1064-1080, 1988.

Ruah CB, Schachern PA, Zelterman D, Paparella MM, Yoon TH. Age-related morphologic changes in the human tympanic membrane. Arch. Otolaryngol. Head Neck Surg. 117:627-634, 1991.

TeOH SW, Flandermeyer DT, Rosowski JJ. Effects of pars flaccida on sound conduction in ears of Mongolian gerbil: acoustic and anatomical measurements. Hear. Res. 106:39-65, 1997.

Uebo K, Kodama A, Oka Y, Ishil T. Thickness of normal human tympanic membrane. Ear Res. Jpn. 19:70-73, 1988.

von Unge M, Borg E, BAgger-SJöBÄck D. The mechano-acoustic properties of the tympanic membrane: a study on isolated Mongolian gerbil temporal bones. Am. J. Otol. 12:407-419, 1991. 